\title{
Constraining kimberlite geology through integration of geophysical, geological and geochemical methods: A case study of the Mothae kimberlite, northern Lesotho
}

\author{
M. Galloway, B. van Coller, T. Nowicki, B. Mukodzani, K. Siemens, C. Hetman, J Gurney, \\ G. Cele \\ Mineral Services Group, Cape Town, South Africa \\ (megan.galloway@minserv.co.za / Fax: +27 (0)21 531-4209 / Phone: +27 (0)21 351-3162)
}

\begin{abstract}
Introduction
The Cretaceous Mothae kimberlite is located in northern Lesotho on the southeast Kaapvaal craton. It is a steep-sided kimberlite pipe emplaced within the sediments and lavas of the Karoo Supergroup. The pipe has an interpreted area of about 8.8 hectares and comprises two lobes, a southern lobe and a smaller northern lobe. An irregular dyke like body is reported to connect the two lobes under thin basaltic cover. Mothae forms part of a field of kimberlites that includes the Liquobong, Kao and Letseng bodies.
\end{abstract}

Basutoland Diamonds Ltd discovered the Mothae pipe in 1961, when a heavy wash of garnets and ilmenites was found downstream from it (Nixon, 1973). Initial reports on grade are provided in publication by Bleackley and Workman in 1963. More comprehensive prospecting was undertaken by Scott | (Meaton, 1966), and later by Lonrho Ltd, between 1969 and 1971 (Nixon 1973). Illegal artisanal miners have been working sporadically at Mothae since the mid 1970's focussing largely on the alluvial material directly downstream of Mothae as well as the residual gravels and weathered kimberlite that are exposed closer to surface in the southwestern portion of the pipe.

Previous evaluation work suggests that Mothae has a low average diamond grade (approximately $3 \mathrm{cpht}$ ), similar to that of the Letseng Diamond Mine, located $6.5 \mathrm{~km}$ to the south-east. Limited historical geological information and bulk sample data provide evidence for significant internal variations within the Mothae pipe and suggest that it may comprise several distinct kimberlite domains of varying diamond content.

Mothae is currently being evaluated by Motapa Diamonds Inc. and its joint venture partner Lucara Diamond Corp. to test the potential for high value diamonds similar to those recovered at Letseng.

\section{Rationale}

Evaluation of the potential for high value diamonds requires excavation and treatment of a large bulk sample (100,000 tonnes) that adequately represents key variations in kimberlite type and grade that may be present. In order to optimise the design of the sampling programme, ground geophysical surveys were undertaken and a grid of pits and trenches was excavated (in accessible portions of the deposit) to better constrain the outline of the pipe and any internal variations in kimberlite type. Material excavated from the pits was examined petrographically and analysed for mantle-derived indicator mineral (KIM) content and whole-rock geochemistry.

\section{Ground geophysics}

A reliable geological framework is an essential starting point for all kimberlite evaluation work. Highresolution ground geophysical surveys can be valuable in providing an early indication of the dimensions of the kimberlite and of major internal geological variations present. The geophysical techniques employed in this study include: (1) a loop-loop frequency domain electromagnetic (EM) technique deployed to highlight the boundary between the conductive kimberlite and relatively resistive wall rock; (2) gravity, which exploits the density contrast between the kimberlite and the wall rock; and (3) magnetics, which utilises the magnetic susceptibility contrast between kimberlite and Karoo basalt. Internal magnetic susceptibility variations within the body may indicate geologically different zones.

The EM survey successfully delineated the kimberlite/basalt contact (Figure 1A). Both vertical loop and horizontal loop configurations indicate a high conductivity body that is continuous between the northern and southern lobes. The total horizontal derivative (THD) of the vertical loop EM indicates internal zones of varying conductivity which may reflect geological variations.

The THD of the Bouger gravity survey defines the kimberlite body convincingly, and correlates closely with the kimberlite/basalt contact defined by the vertical loop EM (Figure 1A). Negative residual anomalies of $\sim 0.6 \mathrm{mgal}$ in the northern lobe and $\sim 0.4 \mathrm{mgal}$ in the southern lobe are seen. The difference in magnitude of these negative anomalies may reflect 
differences in rock type but could also indicate a higher degree of alteration/weathering in the northern lobe.

The total magnetic intensity (TMI) data collected during the magnetic survey indicates two magnetic units corresponding with the northern and southern lobes. Vertical integration of the TMI suggests that the lobes may be continuous at depth. In addition, the southern lobe exhibits distinct internal magnetic variations that can be divided into two zones - a prominent magnetic high in the western portion and an adjacent low in the eastern portion. These may be consistent with different units of kimberlite. Smaller, more localized variations can also be identified, and may also delineate units (Figure 1A).

\section{Petrography and Mapping}

Geological mapping and ground-truthing of kimberlite contacts, as inferred from ground geophysics, were undertaken by means of surface pitting (set out in a rough grid) and limited trenching. The intention of the petrographic work was to establish the character of and variability in kimberlite geology. Detailed characterisation of kimberlite units in terms of key components and textures provides valuable information on the mode of emplacement and factors relevant to the quantity and distribution of diamonds.

Petrographic work indicates that the upper portion of the body is primarily comprised of massive, fragmental volcaniclastic kimberlite with extensive surface weathering obscuring petrographic details. Despite the weathering, geological variations are evident, highlighted primarily by variations in the character and abundance of lapilli, magmaclasts, country rock xenoliths and mantle peridotite xenoliths. Seven distinct kimberlite types (Type I to Type VII) are identified on the basis of these features and their spatial distribution is shown in Figure 1B. Note the correlation between the various petrographic kimberlite types, and the geophysically defined units.

\section{Indicator Mineral Abundances}

The mantle sample in kimberlite can be directly assessed through quantitative evaluation of the abundance and composition of mantle-derived xenocryst minerals (kimberlitic indicator minerals or KIMs).
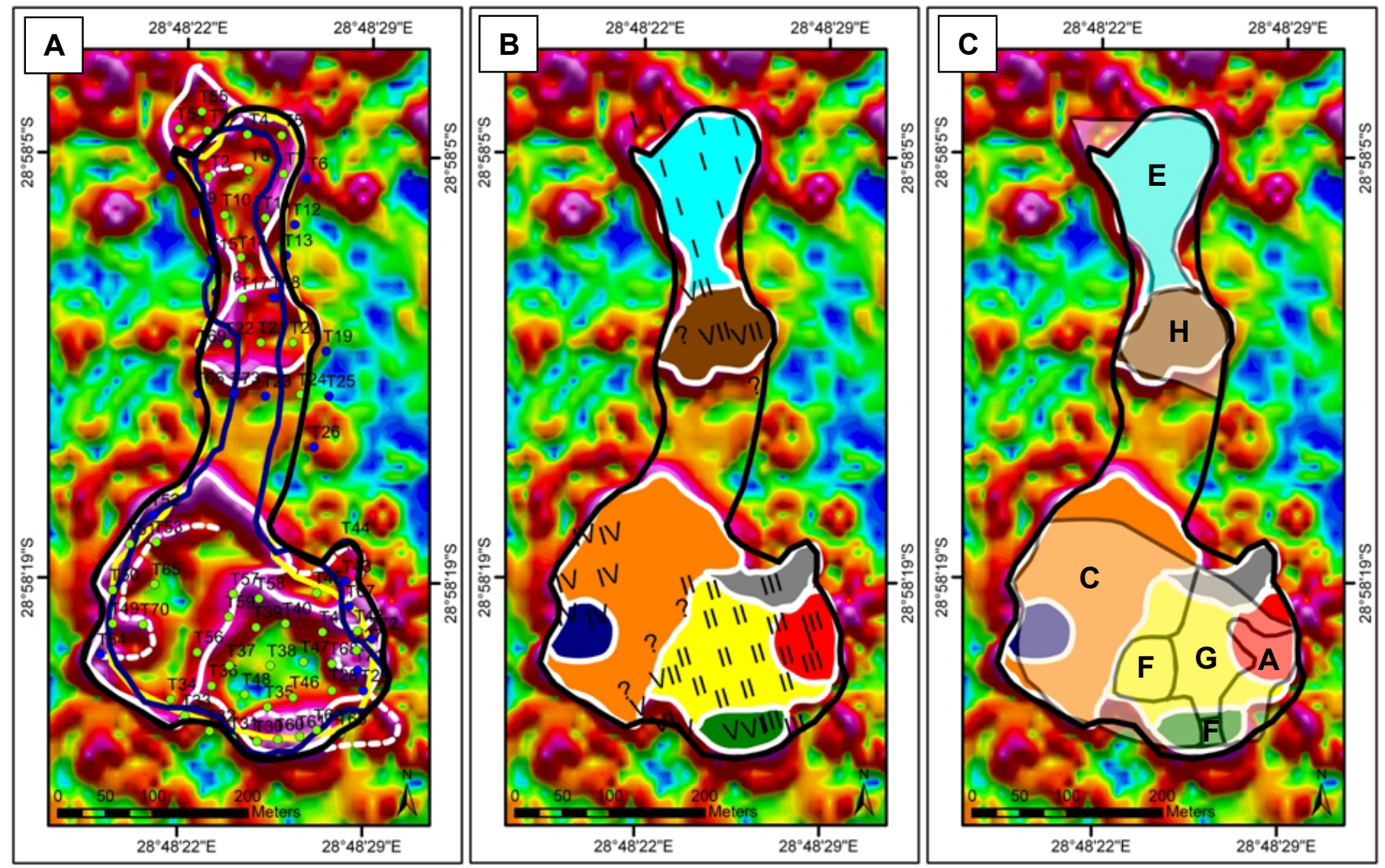

Figure 1. (A). Kimberlite/basalt contacts defined by electromagnetics (blue), gravity (yellow), and magnetics (white) superimposed on the THD of the TMI. Final kimberlite/basalt contact based on all techniques is shown in black.

Internal variations defined by magnetics are shown (white). Sample pits are shown in green and blue, where kimberlite and basalt respectively were intersected. (B). Spatial distribution of the various kimberlite types (I-VII) based on petrographic study superimposed on kimberlite units as defined by magnetics (coloured polygons). (C). Kimberlite domains based on KIM abundances (transparent polygons labelled A-H) superimposed on kimberlite units as defined

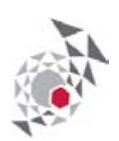
by magnetics (coloured polygons). 
In addition to petrography/mapping done on the pits and trenches, forty-nine rock samples were collected and processed by the Mineral Services standard Mantle Mapper ${ }^{\mathrm{TM}}$ method to determine the absolute abundances of specific varieties of kimberlitic indicator minerals per $\mathrm{kg}$ of rock. The results of this analysis define 6 distinct sample groups, based primarily on the ratios of different garnet types and chromite. An example of these groupings is showing in Figure 2. When plotted spatially, these groups of samples define coherent zones that correlate closely with units defined on the basis of geophysical and geological data (Figure $1 \mathrm{C})$.

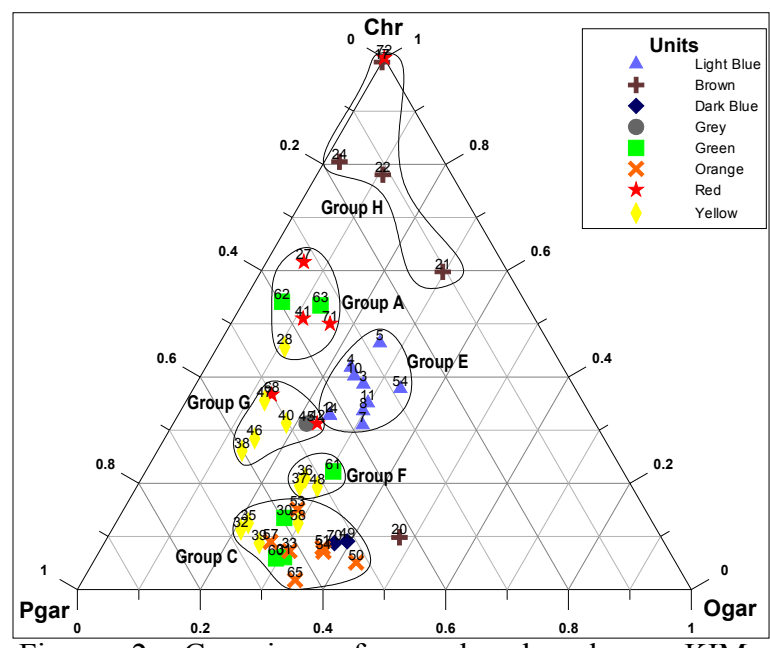

Figure 2. Grouping of samples based on KIM abundances. A. Ternary diagram with apices representing the relative abundances of visually identified mineral grains in samples from the Mothae kimberlite; purple garnet (Pgar), orange garnet (Ogar) and chromite (Chr). Samples are colour coded on the basis of the geophysically-defined unit from which they were derived.

\section{Whole rock geochemistry}

Having defined possible geological domains based on the KIM abundances, the whole rock geochemistry was reviewed to assess whether it could provide support to the model. Aliquots from forty-eight of the samples were submitted to ALS Chemex for quantitative major and trace element compositional analysis.

There are broad similarities in bulk composition between samples from the various defined domains, but there are some key differences that can distinguish between domains. Samples from domain $\mathrm{H}$ are distinct based on their high (and variable) contamination indices (Clement, 1982). The same samples also exhibit high concentrations of several major and trace elements, e.g. $\mathrm{Zn}$ and $\mathrm{Ga}$. Other domains can be distinguished from one another by their $\mathrm{Ni}$ and $\mathrm{Cr}$ contents. In addition, subtle variations in the concentration of other trace elements (Tm, Ce and V) also reveal differences between domains. Key results from the geochemistry work are; (1) geochemical varrations provide further support for subdivision of the pipe based on parameters discussed above; and (2) the details of these variations reflect the nature of the subdivided units. For example, samples with high CI are likely to contain the highest proportion of crustal material whereas elevated $\mathrm{Ni}$ contents may be indicative of increased concentration of olivine and, possibly a higher degree of fines depletion by volcanic processes.

\section{Conclusions}

There is a good correlation between units independently defined on the basis of geophysics, petrography and KIM abundances and these are well supported by geochemical trends. These results are believed to confirm the presence of multiple domains of kimberlite, with different geological characteristics, within the Mothae pipe. These defined zones are likely to reflect emplacement processes which can influence the concentration and distribution of diamonds. This is significant in the evaluation context. In addition, variations in KIM abundance indicate variations in the character of the mantle sample which is directly relevant to the diamond potential of each domain.

Overall, the methods described above delineate four major domains at Mothae - two in the northern section, and two in the southern lobe (east and west). The eastern half of the southern lobe exhibits multiple smaller units where the correlation between different data types is not as precise. These geological subdivisions have been used as a basis for layout of bulk sample pits in such a way to ensure that all potentially significant phases are appropriately represented. In addition, the resultant map of internal geology provides a framework for planning of further work towards ultimately establishing a resource model for the Mothae kimberlite. This study provides an example of how an integrated, multidisciplinary approach can enhance the understanding of complex kimberlite bodies at early stages of investigation and how this may contribute towards their successful evaluation.

\section{Acknowledgements}

Lucara Diamond Corp. and Motapa Diamonds Inc. are thanked for permitting the publication of the information presented.

\section{References}

Bleackley, D. and Workman, D.R., 1963. Reconnaissance mineral survey of Basutoland. Overseas Geological Surveys

Clement, R., 1982. A comparative geological study of some major kimberlite pipes in the Northern Cape and Orange Free State. Unpublished $\mathrm{PhD}$ thesis, University of Cape Town

Meaton, E. St. P., 1966. Basutoland diamonds: Evaluation of selected kimberlites and alluvials. London, Report of the Ministry of Overseas Development.

Nixon, P.h., 1973. The geology of Mothae, Solane, Thaba Putsoa and Blow 13. In Nixon, P.H. (Ed.), Lesotho Kimberlites. Lesotho National Development Corporation. 